\title{
Educación para una ciudadanía global y aprendizaje intercultural: obstáculos y oportunidades ${ }^{*}$
}

\author{
Global citizenship education and intercultural learning: \\ obstacles and opportunities
}

\author{
Francis Mason Bustos ${ }^{* *}$ \\ Departamento de Sociología, Universidad Alberto Hurtado, Santiago, Chile
}

Recibido: 12 de junio de 2015. Aprobado: 16 de septiembre de 2015.

\begin{abstract}
Resumen
La promoción del aprendizaje intercultural ocupa un rol central en la formación de ciudadanos conscientes de los desafíos que implica vivir en una sociedad global e interconectada. En ello se reconoce la necesidad de aprender a dialogar y convivir en la diferencia, fomentando habilidades concretas que permitan el reconocimiento, valorización y entendimiento de la diversidad cultural en sus múltiples formas y contextos. Distintas organizaciones educacionales internacionales responden a los objetivos de una educación para una ciudadanía global brindando oportunidades de aprendizaje intercultural por medio de programas de intercambio intercultural en el extranjero. Sin embargo, en este contexto la relación entre interculturalidad y educación para una ciudadanía global es compleja y marcada por importantes obstáculos. Entre ellos la promoción de culturas nacionales como expresión de diversidad, la minimización de asimetrías de poder en relaciones interculturales, el no reconocimiento de la permanente tensión entre diversas orientaciones normativas sobre la construcción de un mundo más justo y pacífico, y finalmente las limitaciones de promover la diversidad cultural desde la distinción entre lo culturalmente propio y lo ajeno. Reconocer estos obstáculos implica ampliar oportunidades para aprender a vivir juntos, y de no ser tomados en consideración, el logro de los objetivo se verá amenazado.

El presente artículo es resultado de una investigación de magister: "De Experiencia Intercultural a Experiencia Transcultural. Una nueva mirada a los programas de intercambio internacional estudiantil dentro del marco de una educación". Tesis para optar el título de Magister en Sociología, Universidad Alberto Hurtado. Santiago, Chile.

* Sociólogo y Magister en Sociología, Universidad Alberto Hurtado. Académico del Departamento de Sociología de la Universidad Alberto Hurtado, Santiago, Chile. Correo electrónico: fmason@uahurtado.cl
\end{abstract}


Palabras claves: educación para una ciudadanía global, aprendizaje intercultural, transculturalidad.

\begin{abstract}
Intercultural learning is crucial to promote awareness of the challenges of living in an interconnected global society. Creating this awareness and improving intercultural dialogue, as well as the recognition, appreciation and understanding of cultural diversity are some of the main goals of global citizenship education. The relation between global citizenship education and intercultural learning is analyzed here through the example of various international educational organizations that provide intercultural learning opportunities through intercultural exchange programs abroad. However, this relationship is complex and characterized by important obstacles such as the promotion of national cultures as expressions of diversity, minimizing power asymmetries in intercultural relations, non-recognition of the ongoing tension between various normative claims for a more just and peaceful world, and finally the limitations of promoting cultural diversity by taking culture as a concept of inner homogenization and outer separation at the same time. To recognise these obstacles means creating further opportunities for learning to live together, as otherwise the proposed objectives may not be achieved.
\end{abstract}

Keywords: global citizenship education, intercultural learning, transculturality.

\title{
Introducción
}

El contexto global donde vivimos nos plantea inevitables e importantes desafíos que afectan a la humanidad en su conjunto, y por tanto no pueden ser abordados únicamente desde actores nacionales, sino que implican a actores tanto a nivel local, nacional, regional y global. Esto conlleva un replanteamiento de las concepciones tradicionales de ciudadanía definidas a partir del vínculo entre individuos y Estados nacionales, para abrir espacios donde la ciudadanía pueda entenderse también desde una dimensión global y a escala de los desafíos globales del siglo XXI. 
El escenario global donde estos desafíos emergen dan cuenta de una compleja relación de fuerzas económicas, políticas y sociales que han facilitado una permanente movilización transnacional de personas, objetos, ideas, bienes y tecnologías a distintas velocidades, desde distintos puntos de origen, generando una creciente aproximación y tensión entre culturas, sociedades y mercados, así también entre creencias, prácticas e ideologías en relación a otros en el marco de relaciones inequitativas de poder, problemas de equidad, sufrimiento, justicia y gobernanza a escala local y global (Appadurai, 2000; Sorrells, 2010).

Desde comienzos del siglo XXI, y con una velocidad nunca antes vista en la historia de la humanidad, los avances particularmente en tecnología y transporte han permitido tener a personas, familias y amigos, estudiantes, personas de negocios y extraños cada día más cerca los unos de los otros. Sin embargo, de ellos solo unos pocos tienen el privilegio de disfrutar experiencias interculturales a través de viajes, lecturas y turismo, dado que una importante mayoría se ve forzada a emigrar, viajar largas distancias fuera de casa buscando oportunidades para satisfacer sus necesidades básicas de subsistencia, y muchas veces también las de sus familias (Sorrells, 2010; Sorrells y Nakagawa, 2008).

Zygmunt Bauman (1999) presenta este escenario afirmando que los efectos de la globalización a escala humana se encuentran disparmente distribuidos. Para dar cuenta de ello propone la distinción entre turistas y vagabundos desde donde ilustra las importantes diferencias en las imágenes de globalidad y bienestar entre aquellos que disfrutan de los beneficios de la globalización y quienes no.

Para Bauman quienes entran en la categoría de turistas tienen la satisfacción de andar por la vida a voluntad de elegir sus destinos de acuerdo a los planes que dispongan. En cambio los vagabundos son excluidos una y otra vez del lugar que ocupan. Así, los turistas se desplazan por el mundo a su alcance porque es irresistiblemente atractivo, y los vagabundos lo hacen porque el mismo mundo es insoportablemente inhóspito. A partir de esta distinción Bauman argumenta la idea que el acceso a la movilidad global es el más elevado de los factores de estratificación social.

La disparidad en las consecuencias a escala humana de la globalización, esta vez leída desde la propuesta de Ulrich Beck (2002), es determinante para comprender los efectos secundarios o no deseados que surgen producto de la controlabilidad limitada de los cánones dominantes de progreso y desarrollo que hoy definen lo que él llama sociedad de riesgo global. Estos riesgos globales, como los efectos del cambio climático y la destrucción de ecosistemas, la manipulación genética, los riesgos de desastres nucleares, 
la creciente escasez de los recursos que hacen posible proyectar el desarrollo y crecimiento sostenido de las sociedades del futuro -petróleo, gas, minerales, agua y alimentos-, entre otros...

ya no se limitan a lugares y grupos, sino que contienen una tendencia a la globalización que abarca la producción y la reproducción y no respeta las fronteras de los Estados nacionales, con lo cual surgen unas amenazas globales que en este sentido son supranacionales y no específicas de una clase y poseen una dinámica social y política nueva. (Beck, 2002, p. 19)

Siguiendo a Beck, los efectos de estas amenazas globales finalmente implican consecuencias para todos quienes habitamos el planeta. Por esta razón generar conciencia y promover iniciativas concretas de acción que apunten a responder a estos desafíos es clave para el futuro de la humanidad.

En este contexto, la educación para una ciudadanía global (ECG) surge como un enfoque educacional que reconoce la necesidad de brindar las oportunidades y competencias necesarias para reflexionar en torno a los desafíos que implica vivir en una sociedad global e interconectada, donde es fundamental la promoción de nuevas maneras de pensar y actuar sobre las complejas relaciones en temas sociales comunes, ecológicos, políticos, culturales y económicos (UNESCO, 2006; 2010). Explícitamente este enfoque, así como la llamada educación para el desarrollo, educación en derechos humanos, educación para la sustentabilidad, educación para la paz y la prevención de conflictos, educación intercultural, entre otros, buscan "abrir los ojos y mentes de las de las personas a las realidades del mundo globalizado, y despertarlas para que logren un mundo con mayor justicia, equidad y Derechos Humanos para todos" (Carvalho da Silva, 2008, p. 12).

Entre las oportunidades y competencias promovidas en estos enfoques educacionales, la puesta en práctica de la interculturalidad a través del aprendizaje y diálogo intercultural ocupa un lugar central (UNESCO, 2006; 2010; 2014a; 2014b; Santos, 2009; Vodopivec, 2012). Sin embargo, y como se intenta demostrar en este artículo, los puntos de encuentro entre los objetivos de una educación para una ciudadanía global y la promoción de experiencias de aprendizaje intercultural, observados específicamente a partir de la experiencia de participación en programas interculturales en el extranjero, están condicionados por una serie de obstáculos que de no ser tomados responsablemente en consideración terminarán limitando o haciendo inviable esta relación. Reconocer estos obstáculos abre entonces oportunidades para despejar el camino y nutrir la esperanza de 
responder de mejor manera a los objetivos propuestos de cara a los desafíos globales del siglo XXI.

\section{Educación para una ciudadanía global}

Con la promoción de una ciudadanía global se hace referencia explícita a la necesidad de reconocer espacios de formación y acción ciudadana que no se reduzcan a comunidades políticas definidas territorialmente por fronteras asociadas a Estados nacionales (UNESCO 2014a; 2015; Delanty, 2000; Gaveta y Tandon, 2010).

Por un lado, y siguiendo a O’Byrne (2003), con el establecimiento de convenios y tratados internacionales ha aumentado la interdependencia de los Estados, quienes además han cedido parte de su soberanía a organizaciones supranacionales como la Unión Europea, en el actuar del parlamento europeo por ejemplo, y con ello la centralidad de los Estados nacionales respecto de la configuración de una idea de ciudadanía se ha erosionado. Por otro lado, dado el creciente desarrollo de organizaciones, corporaciones y movimientos de la sociedad civil de carácter transnacional, así como el fortalecimiento de marcos internacionales de derechos humanos, el potencial unificador de los Estados nacionales respecto de sus ciudadanos se vuelve también insuficiente.

Para Delanty (2000) esta insuficiencia se justifica en que las concepciones tradicionales de ciudadanía tienden a no reconocer la diversidad cultural dentro de cada Estado nación, y con ello, bajo un supuesto de igualdad, se minimizan las distintas concepciones de bien común que compiten dentro de estos territorios. Ante este problema, los actuales debates respecto del concepto de ciudadanía se centran en los criterios de inclusión y exclusión, no solo en términos de deberes y derechos, sino también en los principios que definen sentimientos de pertenencia a una comunidad política que no se limite al territorio de los Estados nacionales sino que se amplíe a múltiples dimensiones: locales, nacionales, regionales y globales (O’Byrne, 2003; Gaveta y Tandon, 2010).

En este contexto, la ciudadanía global no busca la instauración de un gobierno mundial ni cualquier otra expresión totalizante que imite las lógicas de un Estado nacional a nivel planetario, sino que surge como respuesta ante la creciente conciencia sobre la naturaleza interconectada del mundo donde vivimos (O’Byrne, 2003; Delanty, 2000; Gaveta y Tandon, 2010). Ella se reconoce como producto de una diversidad de proyectos 
de bien común que compiten en el mundo, más que por la institucionalización de marcos normativos propios de los Estados nacionales (Abdi y Shults, 2008). De esta manera, la ciudadanía global no respondería a un estado jurídico sino más bien a una metáfora ética que postula un sentimiento de pertenencia a una comunidad global y a la humanidad en su conjunto, donde quienes la integran comparten valores de solidaridad y una identidad colectiva, así como una responsabilidad común a nivel internacional (UNESCO, 2014a; 2015).

Cuando esta discusión se lleva al plano educativo, la llamada educación para una ciudadanía global (ECG) ocupa un lugar central. La ECG apunta explícitamente a la formación de ciudadanos en un contexto global, donde se reconozca la relevancia de los conocimientos, habilidades y valores necesarios para la participación ciudadana en distintas dimensiones de desarrollo social, económico y cultural vinculadas a un plano local y global. En ello se destaca la promoción del entendimiento, la capacidad de asociación con otras personas para pacíficamente resolver problemas, desarrollar tolerancia ante la diferencia y sobre todo una disposición hacia la cooperación entre diversos actores (UNESCO, 2012; 2014a).

La ECG corresponde a uno de los principales objetivos educaciones para la agenda de desarrollo post-2015 promovida por la UNESCO (2014a; 2015) y tiene como importante antecedente el trabajo de la Comisión Internacional Para la Educación del siglo XXI, quienes presentan en 1996 el reporte Learning: The Treasure Within (Delors et al., 1996) el cual ha guiado la definición de las actuales dimensiones estratégicas de la educación para una ciudadanía global (UNESCO, 2014b; 2015). En este reporte se proponen cuatro pilares fundamentales desde donde se sostiene el modelo educativo definido para enfrentar los desafíos globales del siglo XXI. Estos pilares son Aprender a Conocer, Aprender a Hacer, Aprender a Vivir Juntos y Aprender a Ser (Delors et al., 1996). El pilar Aprender a Vivir Juntos es uno de los principales, ya que integra a los otros tres dentro de un proyecto que reconoce la necesidad de definir caminos de encuentro en la diversidad y así responder juntos a los actuales desafíos globales que afectan a la humanidad (UNESCO, 2014b). Específicamente la iniciativa Aprender a Vivir Juntos implica:

aprender a vivir juntos, mediante el desarrollo de una comprensión de los demás y su historia, las tradiciones y los valores espirituales y, de esta manera, la creación de un nuevo espíritu que, guiados por el reconocimiento de nuestra creciente interdependencia y análisis común de estos riesgos y desafíos del futuro, motivaría a las personas 
a poner en marcha proyectos comunes para gestionar los inevitables conflictos de una manera inteligente y pacífica. (Delors et al., 1996, p. 22) ${ }^{1}$

Desde este marco de referencia la UNESCO activamente promueve iniciativas con miras a la formación de ciudadanos de mundo. La llamada La Educación Ante Todo o UN Global Education First Initiative es una de ellas y ha sido clave en la promoción de una educación de calidad, pertinente y transformadora para todos de cara a la dimensión educacional de los objetivos de desarrollo del mileno. Para ello se propone avanzar en tres ámbitos prioritarios: (a) escolarizar a todos los niños y niñas, (b) mejorar la calidad del aprendizaje y (c) fomentar la conciencia de ser ciudadanos de mundo (UNESCO, 2012).

Respecto del último ámbito, para fomentar la conciencia de ser ciudadanos de mundo se explicitan una serie de medidas. Entre ellas: (a) enseñar valores, conocimientos y competencias necesarias para la paz, la tolerancia y el respeto de la diversidad, (b) cultivar un sentimiento de comunidad y de participación activa para devolver lo recibido a la sociedad y (c) velar porque las escuelas estén exentas de todas las formas de discriminación, incluidas las desigualdades entre los sexos, la intimidación, la violencia, la xenofobia y la explotación (UNESCO, 2012).

Luego de la evaluación de la iniciativa La Educación Ante Todo, y con miras hacia la agenda de desarrollo post-2015, desde la UNESCO se han definido tópicos y objetivos de una educación para una ciudadanía global a partir de tres dominios interrelacionados (2015, p.15) y que aquí presento resumidos:

Dominio cognitivo: apunta a la adquisición de conocimientos, habilidades de entendimiento y pensamiento crítico sobre fenómenos globales, regionales, nacionales y locales, reconociendo la interconexión e interdependencia entre diferentes países y poblaciones.

Dominio socio-emocional: se dirige a la promoción de un sentimiento de pertenencia a una humanidad común, compartiendo valores y responsabilidades, fomentando empatía, solidaridad y respeto a las diferencias y a la diversidad.

$1 \quad$ Traducción propia, la version en inglés: "learning to live together, by developing an understanding of others and their history, traditions and spiritual values and, on this basis, creating a new spirit which, guided by recognition of our growing interdependence and a common analysis of the risks and challenges of the future, would induce people to implement common projects or to manage the inevitable conflicts in an intelligent and peaceful way." (Delors et al., 1996, p. 22). 
Dominio actitudinal o behavioural: apela a fomentar un actuar efectivo y responsable a nivel local, nacional y global buscando formas de construir un mundo más pacífico y sustentable.

Para cada uno de estos dominios se presentan objetivos y atributos específicos de aprendizaje, de manera diferenciada tanto por tópicos como por niveles de educación y edades (UNESCO, 2015, p. 29).

\section{Aprendizaje intercultural y ciudadanía global}

\section{El caso de programas educacionales de intercambio intercultural en el extranjero}

En la medida que aumenta el reconocimiento de que las sociedades nacionales se configuran a partir de diversas formas de comprender ideales de bien común para todas y todos, el referente nacional deja de ocupar un lugar central para aunar sentimientos de pertenencia ciudadana. En ello, la importancia de promover la diversidad se transforma en una demanda clave toda vez que, por un lado, las sociedades nacionales pasan a ser reconocidas como multiculturales y pluriétnicas, y por otro, el concepto mismo de ciudadanía pasa a ser concebido en múltiples niveles: local, nacional, regional y global (Delanty, 2000; Gaveta y Tandon, 2010; O’Byrne, 2003).

Desde la educación intercultural se responde al llamado de la ECG al reconocer en sus bases fundamentales la necesidad de Aprender a Vivir Juntos en la diversidad (UNESCO, 2006). Específicamente la interculturalidad es reconocida como "una forma de interacción equitativa entre diversas culturas y la posibilidad de generar expresiones culturales compartidas, adquiridas por el diálogo y el respeto mutuo" (UNESCO, 2006, p. 17). Al respecto, la pretensión de diálogo se enmarca más allá de la mera conversación entre personas y depende del cumplimiento de una serie de condiciones de interacción específicas, donde apliquen criterios de validez y simetría en términos de relaciones de poder que permitan mantener la dualidad en el seno de la unidad (UNESCO, 2006; Molina, 2002; Sorrells y Nakagawa, 2008). 
Independiente del enfoque empleado en prácticas de educación intercultural, ya sea en temáticas étnicas o aquellas asociadas a fenómenos migratorios de baja y alta calificación, desde el aprendizaje intercultural se reconocen esfuerzos dedicados al aprendizaje efectivo de competencias y conocimientos que faciliten el diálogo y la convivencia con la diferencia, fomentado habilidades concretas que permitan el reconocimiento, valorización y entendimiento de la diversidad cultural en sus múltiples formas y contextos. En este tipo de aprendizaje, el desarrollo de una sensibilidad intercultural así como la adquisición de competencias interculturales específicas que favorezcan el diálogo intercultural se encuentran cada vez tomando mayor protagonismo (Bennett, 1986; 1993; 2004).

La promoción de aprendizaje intercultural y el desarrollo de un sentimiento de pertenencia a una comunidad que trascienda fronteras nacionales son parte importante de las motivaciones que actualmente definen la creciente oferta de programas educacionales de intercambios interculturales en el extranjero. En ellos la apuesta educativa apunta a que durante la participación en un programa de intercambio intercultural los estudiantes aprenden a vivir en la diversidad que implica un nuevo idioma, una nueva familia, una nueva escuela, nuevos amigos y amigas, y una nueva comunidad local en el extranjero.

El mercado de estos programas de intercambio está compuesto principalmente por organizaciones internacionales, voluntarias y sin fines de lucro que surgen a mediados del siglo XX, con directa influencia de la experiencia de sus fundadores en misiones de paz durante la primera y la segunda guerra mundial, con el objetivo de promover programas educacionales y servicios comunitarios orientados hacia el fortalecimiento de la paz, el entendimiento y la cooperación entre personas de diversas culturas. Entre estas organizaciones destacan AFS Programas Interculturales (ex American Field Service), Youth for Understanding (YFU), AIESEC, Experiment in International Living, Rotary Club, entre otras (Mason, 2013).

El concepto de interculturalidad que domina entre estas organizaciones apunta a la relación o interacción entre culturas, considerando más bien a individuos de diferentes orígenes culturales, asumiendo que la cultura es un factor importante a la hora de comprender el comportamiento de los individuos y su visión de mundo. Esto tiene estrecha relación con los postulados de uno de los más influyentes pensadores del campo de la comunicación intercultural, Edward T. Hall (1959), quien afirma que "cultura es comunicación y comunicación es cultura" (p. 186), donde la cultura es considerada por el autor como un sistema de patrones que son aprendidos y son posibles de ser analizados en las 
formas en que nosotros nos comunicamos de manera verbal como no verbal (Collier, 2011).

En ello, las referencias a las condiciones específicas que faciliten el diálogo intercultural se encuentran en el ejercicio de establecer formalmente contactos entre individuos, sin importar el origen cultural ni político, para que en base a experiencias intersubjetivas, como vivir en la cultura de otro país -como estudiante internacional de intercambio o voluntario en servicios comunitarios-, se gatillen oportunidades de aprendizaje intercultural, destacando por ejemplo la relevancia de reflexionar críticamente sobre las consecuencias de realizar juicios y actos etnocéntricos en contextos culturalmente diversos (Ruffino y Chinzari, 2014; Bennett, 2004).

Dentro del mapa conceptual y analítico que le da sentido al trabajo de estas organizaciones de intercambio se reconoce que las personas son portadoras de cultura y así se diferenciarían las unas de las de las otras (Humphrey, 1998). Para explicar el concepto de cultura y las diferencias entre culturas se utilizan diversos modelos explicativos, siendo el Modelo del Iceberg (French y Bell, 1984) uno de los más empleados. En él se define gráficamente a la cultura como un iceberg, en cuya superficie se puede identificar una parte visible, mientras que la otra gran parte queda invisible al estar sumergida bajo el agua. Los elementos de la cultura que se pueden observar, como las formas de comer o vestir, estarían representadas por la parte superior y visible del iceberg, mientras que los elementos no obvios o más profundos de la cultura, como los componentes simbólicos, valóricos, emocionales y cognitivos se encontrarían sumergidos en el agua y por tanto no se pueden ver desde la superficie. Siguiendo esta idea, una experiencia de aprendizaje intercultural en el extranjero implicaría seguir la metáfora de sumergirse y explorar la parte inferior del iceberg que se encuentra bajo el agua durante un periodo de tiempo definido. 


\section{Formación de ciudadanos de mundo desde el aprendizaje intercultural: principales obstáculos}

\section{Promoción de culturas nacionales}

La relación entre aprendizaje intercultural y la promoción de una educación para una ciudadanía global es compleja y un primer obstáculo se encuentra en la manera que se promueve la diversidad cultural.

Producto de un análisis documental de las principales organizaciones que ofrecen programas de intercambio intercultural en el extranjero, se observa una importante tendencia hacia la promoción de la diversidad cultural tomando como referencia ideales de culturas nacionales. Es decir, vivir o sumergirse en la cultura del país de intercambio, ya sea la cultura alemana, la cultura china, la cultura chilena, y así con cualquiera de los países donde sea posible vivir una experiencia de intercambio.

No obstante lo anterior, al momento de promocionar cada país y su cultura, es fundamental preguntarse sobre qué tipo de representaciones se están poniendo en juego cada vez que se define y transmite una idea particular de cultura nacional: preguntarse qué se incluye y qué se excluye, y cuáles son las razones detrás de esta distinción (Sorrells y Nakagawa, 2008).

Dado el carácter internacional de los programas de intercambio, la referencia a trasladar temporalmente a una persona desde su medio ambiente habitual a otro completamente nuevo invita explícitamente a vivir experiencias de aprendizaje intercultural en una cultura nacional localizada geográficamente afuera, reproduciendo ideales de culturas nacionales cargadas de significaciones que la asocian a una unidad culturalmente homogénea, fija en el tiempo, definida permanente en los límites de algún territorio nacional, y por sobre todo, distinta a la cultura propia.

Sin embargo, distintas investigaciones y discusiones académicas confirman que las culturas nacionales, de existir, no serían totalidades homogéneas. Tampoco cuentan con una esencia fija que las defina para siempre en el tiempo, ni menos se presentan como culturas esféricas, aisladas las unas de las otras, como distintos icebergs, o como un archipiélago de islas (Rogers, 2006; Thurlow, Killick, Parry y Phipps, 2001; Welsch, 1999; 
Restrepo, 2014), sino que las culturas nacionales no son más que la alusión a una comunidad imaginada, ya que "aún los miembros de la nación más pequeña no conocerán a la mayoría de sus compatriotas, no los verán ni oirán siquiera hablar de ellos" (Anderson, 1993, p. 23).

Con la promoción de culturas nacionales se realzan imaginarios culturales particulares que buscan representar a toda una nación desde una sola voz dominante, minimizando las disputas por reconocimiento que marcan la diversidad en cada Estado nación (Anderson, 1993; Meinhof y Triandafyllidou, 2006). En este sentido, las referencias a culturas nacionales generalmente responden a la postura de un grupo cultural-político dominante que asume lo nacional como una extensión política de sí mismos, y en último caso, no significan más que una sobre-generalización de lo nacional (Larraín, 2005; 2008; Meinhof, 2009).

Toda referencia a una ciudadanía global sería insuficiente si el canon que domina en la promoción de la diversidad cultural se presenta en términos de culturas nacionales. Esta es la misma razón por la cual el concepto de ciudadanía nacional pierde centralidad y aumentan los llamados para reconocer formas de ciudadanía que superen referentes nacionales y que respondan a la alta diversidad presente dentro de cualquier territorio nacional. Sobre esto último, Renato Ortiz (2014) nos invita a reconocer la diversidad como uno de los principios fundamentales al momento de definir cualquier sociedad nacional. Por esta razón, la diversidad cultural, como rasgo constitutivo y permanente de todos los ámbitos y niveles de la vida social, no sólo se encontraría afuera u en otro país, sino que existe y se vive localmente dentro de cualquier comunidad cultural a la cual se le atribuye un sentimiento de pertenencia.

Formas y expresiones que dan cuenta de esta diversidad se observan por ejemplo desde los lentes que ofrecen las teorías de estratificación social. Desde ahí se reconocen distintos grupos sociales de acuerdo a principios específicos, siendo los más comunes, el nivel de ingreso, rentas y potencial de consumo, así como formas de prestigio, poder y estatus social (Martuccelli, 2007). La importancia de reconocer la diversidad es que en cada uno de estos grupos la realidad social y cultural opera y es significada de manera diferente, independiente de si son o no ciudadanos de un mismo país (Sorrells, 2010).

Minimizar la diversidad interna de cada país promoviendo culturas nacionales es entonces uno de los principales obstáculos a superar si es que desde el aprendizaje intercultural en programas de intercambio en el extranjero se desea realmente contribuir a la formación de ciudadanos de mundo. Para lograrlo es manifiesta la necesidad de ampliar 
el repertorio conceptual y desafiar crítica y responsablemente los conceptos desde los cuales se define y localiza la diferencia, partiendo por reconocer y promover la diversidad de formas en que la vida personal y social es significada dentro de distintos grupos sociales, desde un nivel local hasta el global.

\section{Minimización de asimetrías de poder en relaciones interculturales}

La promoción del reconocimiento, diálogo y el respeto en contextos interculturales son principios fundamentales a la hora de pensar la construcción de sociedades más justas, pacíficas, tolerantes e inclusivas. Desde estos principios se busca proporcionar a la población los conocimientos y valores necesarios para resolver colaborativamente los problemas asociados a los desafíos del siglo XXI. Sin embargo, desde estudios postcoloniales en la discusión latinoamericana, autores como Walsh (2011) y Tubino (2005) problematizan enfoques a favor del diálogo, basados en modelos interculturales y multiculturales, preguntándose ¿a quién es funcional el uso del concepto de interculturalidad?

La crítica emerge como una contra-respuesta a políticas etnoeducativas e interculturales que empezaron a implementarse en América Latina desde los años 90. Estas apuntan al reconocimiento de la diversidad cultural dentro de cada sociedad nacional, donde emerge principalmente la tensión entre culturas indígenas y una cultura nacionalestatal dominante. En ello, si bien desde el Estado se presentan posturas pro diálogo, colaboración y consenso, lo que se propone está lejos de ser un problema simple. Esto ya que las disparidades de poder entre los actores en relación es minimizada deslegitimando de paso cualquier posibilidad de diálogo fecundo y en igualdad de condiciones.

Esta discusión adquiere fuerza desde las ideas de Stuart Hall (1997) quien postula que la cultura es un campo de disputa de significados, donde existen diferencias en las relaciones de poder y donde unos adquieren el carácter de dominantes y otros de dominados, excluidos o silenciados, lo cual corresponde a un problema que no se reduce únicamente a relaciones interculturales a nivel nacional, sino que es un problema escalable globalmente (Sorrells y Nakagawa, 2008; Sorrells, 2010; Zizek, 1998; De Sousa Santos, 2003).

Con estos antecedentes, la promoción del aprendizaje intercultural sin dar a conocer enfática y responsablemente que durante relaciones interculturales los sujetos pueden 
encontrarse en situaciones de asimetrías de poder y privilegios es más un obstáculo que un facilitador del diálogo fecundo, la colaboración, la búsqueda del consenso. Por esta razón, sumada al sesgo de promover ideales de culturas nacionales, cada vez que se omite o se le resta importancia a la dimensión que da cuenta de las disparidades de poder en una relación intercultural, se potencia el peligro de invisibilizar y minimizar los factores que pueden haber gatillado las situaciones históricas de conflicto, reproduciendo nuevamente diferencias de poder, disparidades y desigualdades.

Este obstáculo adquiere especial relevancia al preguntarnos sobre el grupo social al que pertenecen aquellos que en su mayoría participan de programas de intercambio intercultural en el extranjero. Siguiendo a Bauman (1999) y Sorrels (2010), estos se encontrarían dentro del grupo de personas privilegiadas que pueden disfrutar los beneficios de la globalización y obtener provecho de la interculturalidad por medio de viajes, lecturas y turismo como un ejercicio de poder, más aun considerando que participan de programas educacionales prestigiosos y reconocidos internacionalmente dada la épica moral que proyectan con la pretensión de formar a ciudadanos de mundo que trabajen por la construcción de un mundo más justo y pacífico.

\section{Tensión entre diversos tipos de cosmopolitismos: universalismo y particularismo}

Cada vez que se hace referencia a valores universales sobre cómo y hacia dónde orientar la acción frente a desafíos globales, queda abierta la pregunta sobre la manera en que una forma particular de entender un mundo mejor adquiere un carácter universal y se posiciona como tal. En este sentido, trabajar para la construcción de un mundo más justo y pacífico implica adoptar una disposición particular sobre el vivir en el mundo, una disposición particular definida a partir de un referente normativo, un deber ser con aspiraciones universalistas y pretensiones de legitimidad.

No obstante lo anterior, existen diversas formas particulares de comprender universales como la paz, democracia, el ejercicio de ciudadanía y la igualdad, por lo que la adopción de una pretensión universalista sobre otra implica siempre una toma de posición normativa particular. La razón radica en que la inagotable diversidad sociocultural del mundo no puede ser reducida a un único canon universalista sin con ello minimizar e invisibilizar otras perspectivas particulares sobre un mundo mejor para todas y todos, por lo tanto, las opciones de tomas de posición son siempre diversas (Ortiz, 2014). 
La tensión entre particulares con pretensiones universales se expresa en el debate académico sobre la tensión entre universalismo y particularismo (Chernilo, 2010) o universalismo y diversidades (Ortiz, 2014), donde se reconoce que esta tensión es permanente y de su resolución, posible o no, depende la definición del camino a seguir pensando en el futuro del mundo.

Renato Ortíz (2014) aborda esta tensión reconociendo que en la sociedad global donde vivimos lo diverso se convierte en el bien común, y por tanto, la exigencia de reconocimiento, principalmente en situaciones de exclusión y discriminación, se convierte en una demanda universal. Es decir, tal como se postula en los lineamientos de una educación para una ciudadanía global, la valorización y el reconocimiento de las diferencias es una de las bases para construir un mundo más pacífico y sustentable.

Con este fin, la importancia de reconocer y valorar las diferencias implica aceptar que "aunque integrado en un mercado global, interconectado por tecnologías de comunicación, el mundo nada tiene de homogéneo" (Ortiz, 2014, p. 28), y para entender esto es clave preguntarse sobre el origen de estas diferencias y reconocer que todas son producidas socialmente y portan un sentido histórico. En este sentido, las relaciones entre particularismos diversos no tienen nada de arbitrario sino que más bien son productos de disputas y conflictos manifiestos en situaciones históricas determinadas, donde las diferencias en las relaciones de poder se reflejan en desigualdades, sobre todo en la innegable asimetría entre países, clases sociales y etnias.

Las diversas formas particulares de abordar universal y normativamente la dirección del devenir del mundo se encuentran fuertemente desarrolladas en el debate sobre el concepto de cosmopolitismo y sus diversos tipos:

En principio, el concepto de cosmopolitismo apela normativamente a la unidad fundamental de la especie humana, e igualdad última de todos los seres humanos, por lo que "las diferencias de género, étnicas, culturales, nacionales y religiosas deben ser teorizadas como algo interno a la unidad sustantiva de la humanidad" (Chernilo, 2010, p. 10). Sin embargo, Alejandro Pelfini (2013) nos indica que la diversidad de formas de vida social humana no puede ser contenida en una única forma de acción con pretensiones cosmopolitas particulares, sino que, más la diversidad se ve reflejada en la tensión entre distintas formas de cosmopolitismos (en plural). Esta tensión, según Gustavo Lins (2003), radica en lo identificable como la fuerza inclusiva en el fomento de una solidaridad universal, lo que implica que, por ejemplo, los cosmopolitismos populares sean 
distintos a los de corporaciones transnacionales, que por su parte difieren también del de turistas burgueses, magnates de los negocios o académicos internacionales.

Al respecto, la propuesta de Pelfini (2013) describe tipos de cosmopolitismos, principalmente haciendo una distinción entre lo que llama cosmopolitismo atlántico y cosmopolitismo minimalista. El primero se caracteriza por representar la propagación de la imagen dominante de globalidad proyectada por los países de Europa occidental y Estados Unidos, mientras que el segundo, el cosmopolitismo minimalista, integra las diversas formas de entender lo universal de la humanidad, donde el cosmopolitismo atlántico sería solo una versión particular más entre otras. En este sentido,

la posibilidad de desarrollar una perspectiva cosmopolita descansa en el reconocimiento de nuestra propia vulnerabilidad (así como la del entorno y de otros seres vivos) respecto de las acciones de los otros, de los peligros de la naturaleza y -last, but not least- de los riesgos generados por el mismo progreso tecnológico. El no herir al otro se convierte entonces en el imperativo fundamental de un cosmopolitismo minimalista. (Pelfini, 2013, p. 18)

En la misma línea argumentativa, Homi K. Bhabha (2013) presenta la distinción entre dos formas de pensamiento cosmopolita que se encuentran profundamente arraigadas en los discursos contemporáneos acerca de la globalización: cosmopolitismo global y cosmopolitismo vernáculo.

Siguiendo a Bhabha (2013), el cosmopolitismo global se define como cosmopolitismo de prosperidad y privilegios relativos, fundados en las ideas de progreso que guardan complicidad con formas de gobierno neoliberales y las competitivas fuerzas del libre mercado. Este concepto de desarrollo global confía en los poderes virtualmente ilimitados de la innovación tecnológica y las comunicaciones globales. Mientras elogia las "culturas del mundo" o "los mercados del mundo", esta clase de cosmopolitismo se mueve con rapidez y de manera selectiva de una isla de prosperidad a otro nuevo terreno de productividad tecnológica, prestando poca atención a la desigualdad persistente y la pauperización que produce este tipo de desarrollo.

Por otro lado, el concepto de cosmopolitismo vernáculo refiere a ideales de progreso desde la perspectiva de las minorías, donde los reclamos de igualdad y libertad están basados en un derecho a la diferencia en la igualdad. Estas agrupaciones o solidaridades minoritarias se plantean en respuestas a las fallas y los límites de la representación democrática, y crean nuevos modos de agencia, nuevas estrategias de reconocimiento, 
nuevas formas de representación política y simbólica, tales como las ONG, los grupos antiglobalización, las comisiones por la verdad, las cortes internacionales, las agencias locales y los tribunales de transición (2013, p. 94-99).

La tensión entre estos diversos tipos de cosmopolitismos es por consiguiente tanto un aporte como un obstáculo para responder a los objetivos de una educación para una ciudadanía global. Es un aporte ya que da cuenta que cada pretensión universalista con miras a la construcción de un mundo más justo y pacífico es siempre una postura particular dentro de otras posibles. Esto permite transparentar y abrir espacios para sincerar la discusión y responder preguntas como ¿qué imagen de globalidad cosmopolita se proyecta desde la promoción de una educación para una ciudadanía global? ¿Es una ciudadanía global que representa a aquellos que entran en la categoría de turistas globales, beneficiados de la globalización, o integran también las posturas y voces de grupos minoritarios, vagabundos, desfavorecidos ante los avances de la globalización?

Como obstáculo la tensión entre diversos tipos de cosmopolitismo emerge en la medida que los dos obstáculos anteriores, a saber, la promoción de culturas nacionales y la minimización de asimetrías de poder en relaciones interculturales, no sean responsablemente tomados en consideración. La razón se encuentra en la necesidad de reconocer y promover la diversidad por sobre la homogenización y generalización. En el caso de la tensión entre cosmopolitismos, la necesidad de promover la diversidad, y no solo aprender de ella y sino también en ella, deja de centrarse en un nivel nacional para responder a interrogantes particulares con pretensiones normativas universalistas que se encuentran tensionadas en el marco de relaciones inequitativas poder en un nivel global. La superación de este obstáculo implica entonces su reconocimiento como parte constitutiva e ineludible del desafío de aprender a vivir juntos de cara a los desafíos globales que como humanidad nos enfrentamos durante el presente siglo XXI.

\section{Promoción de las relaciones interculturales desde la distinción entre lo cultural- mente propio y lo ajeno: separatismo y exotización de la diferencia}

Este cuarto obstáculo emerge desde la reflexión crítica sobre las ventajas y desventajas del concepto de cultura en el marco de una educación intercultural con miras a la formación de ciudadanos de mundo (Mason, 2013). 
En primera instancia, cuando se afirma que la interculturalidad responde a una forma de interacción equitativa entre diversas culturas (UNESCO, 2006; Molina, 2002), la forma de comprender qué se dice cuando se dice cultura no deja de ser altamente compleja (Güell, 2008). En el campo de la comunicación intercultural se promueve un concepto de cultura entendido como un sistema de patrones que son aprendidos y pueden ser estudiados en las formas en que las personas se comunican con otros (Collier, 2011). Desde esta perspectiva la interculturalidad es analizada en la relación entre sujetos provenientes de diversas culturas que se encuentran y que verbal como no verbalmente se comunican, y de esto último dependerían entonces las posibilidades de entenderse y dialogar pacíficamente.

La principal crítica que surge a esta forma de abordar las relaciones interculturales apunta específicamente a la presunción de que un individuo pertenece de manera fija y para toda la vida a una sola cultura que lo define, y por tanto la comunicación intercultural se realizaría única y exclusivamente desde la pertenencia a esta cultura (Rogers, 2006; Schmidt-Welle, 2011; Welsh, 1999; 2011).

En este contexto, las interrogantes sobre lo que representa cada cultura y su influencia en las formas de comunicación de las personas son claves para comprender los alcances de cualquier relación intercultural. Al respecto, siguiendo el trabajo de Wolfgang Welsh $(1999 ; 2011)$ y Friedhelm Schmidt-Welle (2011), el principal problema se encuentra en que la identificación de una cultura como propia y a la vez distinta a otra considerada como ajena o extranjera es sesgada. Este sesgo indica en gran medida que la noción de interculturalidad seguiría aferrándose en la idea de que la culturas tienen componentes que les son propios y auténticos como características inherentes y esenciales a su ser, y por consecuencia, todo lo demás le es ajeno, siendo esto no otra cosa que pura ficción. De esta manera las consecuencias de promover relaciones interculturales desde el sesgo entre lo propio y lo ajeno ahondaría en la exacerbación de las diferencias culturales desde puntos de vistas separatistas y exotizantes, lo cual implica importantes limitaciones a la hora de promover el entendimiento, capacidad de asociación y combate a distintas formas de discriminación.

Para responder a este sesgo vale destacar que las culturas están lejos de ser totalidades homogéneas y diferentes las unas de las otras (Anderson, 1993; Meinhof y Triandafyllidou, 2006), sino que entre ellas existen transversalmente múltiples fuentes de sentido que se encuentran en constante movimiento (Rogers, 2006) y que son subjetivadas diversamente por los individuos dependiendo de su propia experiencia en el mundo, la posición que ocupen dentro de la estructura de poder, y también del rol que histórica- 
mente han ocupado en los procesos de producción social de las diferencias y disputas por sus reconocimientos.

Estas fuentes de sentido que fluyen a través de las múltiples fronteras son reflejo de fenómenos transnacionales, donde personas, prácticas, ideas, información, bienes y recursos viajan de manera tal que configuran campos sociales que cruzan transversamente a los Estados nacionales, imposibilitando con ello concebir lo local como algo estático, fijo e inamovible (Basch, Schiller, y Blanc, 1994; Bauman, 1998; 1999; Appadurai, 2000). Por esta razón es que no es posible encontrar aquello que marque el origen auténtico de una cultura en particular, sino que "hoy ya nada es totalmente propio ni totalmente extranjero" (Welsch, 1999, p. 2, traducción propia).

Richard Rogers (2006) a partir del concepto de apropiación cultural da cuenta del carácter relacional de las culturas al analizar distintas formas en que éstas se interconectan y se van influenciando mutuamente a través del intercambio cultural, formas de dominación cultural y explotación cultural.

Siguiendo a Rogers (2006), una primera forma de entender la apropiación cultural es el intercambio cultural. Este se realiza en términos de reciprocidad de símbolos, artefactos, rituales y tendencias entre representaciones culturales diferentes que se relacionan dentro de un marco de igualdad de relaciones de poder.

Una segunda forma de apropiación cultural, siguiendo al mismo autor, es la dominación cultural. En este caso la apropiación cultural se establece en importantes asimetrías de poder. Acá el uso de patrones culturales, símbolos y valores de una cultura dominante son impuestos a la fuerza en miembros de otra cultura con características de subordinación.

Consecutivamente, una tercera forma de apropiación cultural es la llamada explotación cultural. Es decir, el uso de elementos de una cultura subordinada por una cultura dominante sin compensación o reconocimiento. Un ejemplo corresponde al uso y adopción de ciertos nombres, conceptos, imágenes, y cualquier otro elemento de una cultura en situación de dominación por miembros de la cultura dominante. Ya sea para nombrar bienes de consumo, realizar campañas de marketing y con ello reconfigurar el significado original del símbolo en disputa.

Tanto en los intercambios culturales, la dominación cultural y explotación cultural se continúa apelando a la relación dual entre culturas. Sin embargo, como lo indica el 
autor, las culturas son producto de la integración simultánea de estas distintas formas de apropiación cultural (Rogers, 2006). Cada una representa una fuente de sentido donde son múltiples los actores involucrados, y por tanto, limitadas las posibilidades de reconocer lo culturalmente propio desde una pretensión de autenticidad y exclusividad (Welsh, 1999; 2011; Schmidt-Welle, 2011).

Con estos antecedentes se hace patente la necesidad de ampliar la batería de conceptos asociados a la cultura en el marco de una relación intercultural y partir por reconocer el carácter relacional que sería transversal a cada una. En este sentido, una relación intercultural pasaría a adquirir un carácter más que inter, asumiendo la relación entre otros diferentes y separados entre sí, como trans, donde la relación se establecería entre diferentes también, pero con la salvedad de que esta diferencia es sólo una parte de la relación, dado que los vínculos, mezclas y encuentros entre estas diversidades son siempre también parte constitutiva de la misma: en ello radica la posibilidad del mutuo reconocimiento.

Desde lo trans, el concepto que surge como alternativa apela a la transculturalidad, donde adoptando las ideas de Wolfgang Welsh (1999), en una relación entre culturas,

el trabajo es reajustar nuestro compas interno: alejándonos de una concentración en la polarización entre los propio y lo externo, y que el reajuste apunte a lo que puede ser común o conectado con aquello externo o extranjero donde sea que se encuentre. (1999, p. 6, traducción propia)

Desde el concepto de transculturalidad no se enfatiza únicamente aquello que marca las diferencias entre culturas, sino que asume que están siempre interrelacionadas y por tanto cuentan con espacios de encuentro, mezcla y similitud entre ellas, espacios que permitirán el mutuo reconocimiento, donde las diferencias y particularidades son manifiestas y defendidas, pero a la vez situadas en un marco híbrido de identificación asumiendo que las culturas, más allá de sus denominaciones, no serían totalmente diferentes las unas de las otras. Welsh (2011) lo explica de la siguiente manera:

De facto, nuestras culturas ya no son homogéneas ni separadas, sino que se complementan, y en gran parte se mezclan. Trato de comprender esta nueva estructura mediante el concepto de la "transculturalidad". Con este concepto quiero llamar la atención sobre el hecho de que la constitución actual de las culturas se encuentra -siguiendo el doble sentido de la palabra latina "trans"- más allá de la vieja (supuestamente esférica) constitución de la cultura. Hoy día, los determinantes culturales pasan 
por y entre las culturas, por lo cual estas últimas ya no se caracterizan por su delimitación evidente, sino por el entretejimiento y su comunidad. (Welsh, 2011, p. 14)

Las descripciones y discusiones sobre procesos de transculturación de las sociedades del mundo dan cuenta del carácter relacional de las culturas (Ortiz F., 1987; Ianni, 2000; Rogers, 2006; Welsh, 1999; 2011; Schmidt-Welle, 2011; Rodríguez, 2010). De esta manera, los objetivos de una educación para una ciudadanía global, entendidos como el fomento de una orientación hacia la cooperación, entendimiento y tolerancia a la diferencia, sobre todo el fomento de un sentimiento de pertenencia a una humanidad común, estarían siendo obstaculizados toda vez que no se adopte y promueva una vigilancia crítica sobre el carácter estático, separatista y exotizante que subyace al concepto de interculturalidad promovido desde la distinción entre lo culturalmente propio y lo ajeno. Por lo tanto, superar de este obstáculo, reconociendo el potencial de la transculturalidad, se transforma en una oportunidad acorde con los desafíos que implica vivir en una sociedad global, interconectada e interdependiente.

\section{Conclusiones}

Los esfuerzos dirigidos hacia la adquisición de conocimientos, habilidades de entendimiento, diálogo y pensamiento crítico sobre fenómenos globales, regionales, nacionales y locales, partiendo por estimular el reconocimiento de la interconexión e interdependencia entre diferentes países, poblaciones y culturas; las acciones orientadas a promover un sentimiento de pertenencia a una humanidad común, fomentando la empatía, solidaridad y respecto a la diferencia; y el fomento de un actuar efectivo y responsable en múltiples niveles con miras hacia la construcción de un mundo más pacífico y sustentable responden a los objetivos de una educación para una ciudadanía global.

El concepto de ciudadanía deja de centrarse en los límites definidos por las fronteras de los Estados nacionales, y por medio de la definición de sentimientos de pertenencia a comunidades políticas desde un nivel local, nacional, regional y global, se abren espacios para comprender formas de acción y participación ciudadana ante temáticas que responden a cada uno de esos niveles. A un nivel global, los desafíos globales a los que se enfrenta la humanidad en su conjunto, como el calentamiento global y los grandes movimientos de personas que este generaría, la erosión del ecosistema, la contaminación ambiental que actualmente hace que el aire de grandes ciudades del mundo sea casi irrespirable, los riesgos de desastres nucleares como el de Chernóbil o Fukushima, la creciente 
escasez de los recursos que hacen posible proyectar el desarrollo y crecimiento de las sociedades en el futuro - petróleo, gas, minerales, agua, alimentación sana-, así también la proliferación de la violencia y organizaciones criminales transnacionales, nos presentan un escenario de crisis donde la necesidad de un cambio resulta imperativa (Beck, 2002; Convivialist Manifesto, 2014; Inge Kaul, 2013; Lenger, Schneickert y Schumacher, 2010).

Desde la promoción de una ciudadanía global se reconoce la necesidad de este cambio apelando a la formación de un sentimiento de pertenencia a una comunidad global, al reconocimiento a una humanidad común y desde ahí a la motivación de acciones responsables que permiten proyectar los cambios necesarios de cara a los desafíos globales del presente siglo. En este sentido, aprender a vivir juntos es central.

Desde la educación intercultural, específicamente desde la promoción del aprendizaje intercultural, se busca promover oportunidades para que personas adquieran las competencias necesarias para efectivamente aprender a vivir juntos, reconociendo nuestra diversidad, y así aprender a dialogar y convivir en la diferencia, fomentando habilidades concretas que permitan el reconocimiento, valorización y entendimiento de la diversidad cultural en sus múltiples formas y contextos, facilitando así las posibilidades de fortalecer encuentros entre personas de diversas partes del mundo que participen activamente en la construcción de un mundo más justo y pacífico. Por esta razón la relación entre el aprendizaje intercultural y una educación para una ciudadanía global es de fuerte cercanía.

No obstante, más allá de la cercanía, esta relación es también altamente compleja y marcada por distintos obstáculos que dificultan el logro de los objetivos propuestos, y que de no ser tomados en consideración se corre el riesgo de reproducir las condiciones históricas que gatillan los conflictos.

La promoción de culturas nacionales como expresión de diversidad es un obstáculo a superar. La minimización de las asimetrías de poder, inequidades y desigualdades toda vez que se hace referencia a una relación intercultural orientada hacia el diálogo y el entendimiento es otro obstáculo a superar. Entre ambos se establece la necesidad de mantener un espíritu crítico a la hora de definir lo que se entiende y promueve como cultura, procurando evitar toda expresión totalizante y homogeneizadora que no dé espacio al reconocimiento de las distintas expresiones culturales que compiten dentro de toda comunidad de sentido donde los individuos establezcan sentimientos de pertenencia. 
La tensión entre universalismo y particularismo, trabajada en la discusión sobre distintos tipos de cosmopolitismos, es reconocida tanto como un aporte como un obstáculo para la consecución de los objetivos de una educación para una ciudadanía global como de una educación intercultural. Su adopción como aporte u obstáculo dependerá de cuán aprehendidos se encuentren los obstáculos relativos a la promoción de culturas nacionales como expresión de diversidad y la minimización de las asimetrías de poder en el marco de relaciones interculturales, ya que si bien la tensión entre tipos de cosmopolitismos es permanente, es también reflejo de la propia diversidad de formas particulares en que se definen horizontes normativos universalistas que apelan a la humanidad como un solo gran actor de cara a la construcción de un mundo más justo y pacífico. Esta diversidad es parte del contexto donde la necesidad de aprender a vivir juntos tiene sentido.

Finalmente, un obstáculo que subyace a los anteriores apela a reconocer las ventajas y las limitaciones del concepto de cultura con que se promueve el aprendizaje intercultural. En los casos donde la cultura y las diferencias culturales son enseñadas a partir de la distinción entre lo propio y lo ajeno, apelando a principios de autenticidad y propiedad originaria, el reconocimiento de la diferencia como premisa básica para aprender a vivir juntos se realizaría desde disposiciones centradas en extrapolar la diferencia, formando fronteras simbólicas rígidas, exotizando la diferencia, entendiéndola como ajena o extranjera, separada a la propia como dos iceberg diferentes o como reflejo de un archipiélago de islas. En estos casos la posibilidad de reconocerse en la diferencia, en un otro distinto, encontrar espacios de encuentro en la diversidad y así fomentar la colaboración y mutuo entendimiento se limita. La salida propuesta aquí es romper con toda idea que promueva a las culturas como contenedoras de una esencia fija, homogénea y estática en el tiempo y espacio, y adoptar una perspectiva que asuma el carácter intrínsecamente relacional de las mismas, apelando por entenderlas como producto de transformaciones históricas y de influencias múltiples entre fuentes de sentido que fluyen transversalmente en el planeta, superando siempre los límites de cualquier frontera nacional.

El paso desde una concepción de interculturalidad basada en la distinción entre lo culturalmente propio y lo ajeno a una concepción que resalte no solo la diferencia sino que también, y principalmente, las dimensiones que dan cuenta de nuestra naturaleza común, entrelazamientos y puntos de encuentro en la diversidad, tanto lo inter como lo trans en las relaciones entre culturas, es una alternativa para fomentar la conciencia respecto de la realidad interconectada del mundo donde vivimos y así también sentimientos de pertenencia que se encuentran en los objetivos de una educación para una ciudadanía global. 
"Ciudadanos del mundo, uníos" aclama Beck (2002, p. 28) para finalizar el manifiesto cosmopolita con el cual introduce su obra llamada "La sociedad del riesgo global". Sin embargo, aprender a vivir juntos, uníos, y seguir el camino para responder a este llamado no está ausente de importantes obstáculos, que más vale conocer.

\section{Referencias}

Abdi, A. y Shultz, L. (2008). Educating for human rights and global citizenship. New York, Estados Unidos: State University of New York.

Anderson, B. (1993). Comunidades imaginadas. Reflexiones sobre el origen y la difusión del nacionalismo. México DF, México: Fondo de Cultura Económica.

Appadurai, A. (2000). Globalization. Carolina del Norte, Estados Unidos: Duke University Press.

Basch, L., Schiller, N. y Blanc, C. (1994). Nations unbound. Transnational projects, postcolonial predicaments, and deterritorialized nation-states. Reino Unidos: Psychology Press.

Bauman, Z. (1998). On glocalization. Or globalization for some, localization for some others. Thesis Eleven, 54 (1), 37-49.

-_-—. (1999). La globalización: consecuencias humanas. Buenos Aires, Argentina: Fondo de Cultura Económica.

Beck, U. (2002). La sociedad del riesgo global. Madrid, España: Ediciones Siglo XXI.

Bennett, M. (1986). A developmental approach to training for intercultural sensitivity. International Journal of Intercultural Relations, 10 (2), 179-196.

- - - . (1993). Towards ethnorelativism. A developmental model of intercultural sensitivity. En M. Paige (ed.), Education for the Intercultural Experience (2nd ed., pp. 21-71). Yarmouth, ME: Intercultural Press.

-———. (2004). Becoming interculturally competent. En J. Wurzel (ed.),Toward multiculturalism: a reader in multicultural education (2nd ed., pp. 62-77). Newton, MA: Intercultural Resource Corporation. 
Bhabha, H. (2013). Nuevas minorías, nuevos derechos. Notas sobre cosmopolitismos vernáculos. Buenos Aires, Argentina: Ediciones Siglo XXI.

Carvalho da Silva, M. (coord.) (2008). Pautas para una educación global. Conceptos y metodologías sobre educación global para educadores y responsables de políticas en materia educativa. España: Centro Norte-Sur del Consejo de Europa.

Chernilo, D. (2010). Nacionalismo y cosmopolitismo. Ensayos sociológicos. Santiago, Chile: Ediciones Universidad Diego Portales.

Collier, A. (2011). How E.T. hall shaped the field and remains relevant today. AFS Intercultural Link, 2 (1).

Convivialist Manifesto. (2014). Convivialist Manifesto. A declaration of interdependence. Duisburg. (Global Dialogues 3). Käte Hamburger Kolleg / Centre for Global Cooperation Research.

De Sousa Santos, B. (2003) Reconhecer para libertar. Os Caminhos Do Cosmopolitismo Multicultural, vol. 3. Brasil: Civilizalccão brasileira.

Delanty, G. (2000). Citizenship in a global age. Buckingham, Inglaterra: Open University Press.

Delors, J. et al. (1996). Learning. the treasure within. Report to UNESCO of the International Commission on Education for the Twenty-First Century. Paris, Francia: UNESCO.

French, W. y Bell, C. (1984). Organization development. behavioral science interventions for organization improvement. New Jersey, Estados Unidos: Prentice-Hall Englewood Cliffs, NJ.

Gaventa, J. y Tando, R. (2010). Globalising citizens: New dynamics of inclusion and exclusion. Londres, Inglaterra: Zed Books.

Güel, P. (2008). ¿Qué se dice cuando se dice cultura? Notas para el nombre de un problema. Revista de sociología, 22.

Hall, E. (1959). The silent language. New York, Estados Unidos: Doubleday New York.

Hall, S. (1997). Representation. cultural representation and signiflying practices. Londres-California, Estados Unidos-Inglaterra: Thousand Oaks Sage. 
Humphrey, D. (1998). Culture as noun, culture as verb, national culture or individual culture. Which approach? En D. Killick y M. Parry (eds.), Languages for cross-cultural capability. Promoting the discipline. Marking boundaries \& crossing borders. Proceedings of the 3rd Cross-cultural Capability Conference. Leeds, Reino Unido: Metropolitan University.

Ianni, O. (2000). Enigmas de la modernidad-mundo. México DF., México: Ediciones Siglo XXI.

Kaul, I. (2013). Global public goods. A concept for framing the post-2015 agenda? Discussion paper 2. Bonn, Alemania: Deutches Insititute für Entcicklungspolitik.

Larraín, J. (2005). ¿América Latina Moderna?. Globalización e identidad. Santiago, Chile: LOM Ediciones.

-_-_. (2008). El concepto dei dentidad. Revista FAMECOS. Mídia, Cultura e Tecnologia, $1(21)$.

Lenger, A., Schneickert, C., y Schumacher, F. (2010). Globalized national elites. Transcience. A Journal for Global Studies, 1 (2), 85-100.

Lins, G. (2003). Postimperialismo. Cultura y política en el mundo contemporáneo. Barcelona, España: Gedisea.

Martuccelli, D. (2007). Cambio de rumbo. La sociedad a escala del individuo. Santiago, Chile: LOM ediciones.

Mason, F. (2013). De Experiencia Intercultural a Experiencia Transcultural. una nueva mirada a los programas de intercambio internacional estudiantil dentro del marco de una educación. Tesis para optar el título de Magister en Sociología, Universidad Alberto Hurtado. Santiago, Chile.

Meinhof, U. H. (2009). Transnational flows, networks and "transcultural capital. Reflections on researching migrant networks through linguistic ethnography”. En J. Collins, M. Baynham y S. Slembrouck (eds.), Globalization and language in contact. scale, migration, and communicative practices (pp. 148-169). Londres, Inglaterra: Continuum International Publishing Group. 
Meinhof, U. H. y A. Triandafyllidou. (2006). Beyond the diaspora. Transnational practices as transcultural capital. En U. H Meinhof y A. Triandafyllidou, Transcultural Europe. Cultural Policy in a Changing Europe (pp. 200-222). Londres, Inglaterra: Palgrave Macmillan.

Molina, F. (2002). Sociología de la educación intercultural. Investigación y docencia en el espacio europeo de educación superior. Buenos Aires, Argentina: Lumen.

O’Byrne, D. (2003). The dimensions of global citizenship: political identity beyond the NationState. Londres, Reino Unido: Frank Cass.

Ortiz, F. (1987). Contrapunteo cubano del tabaco y el azúcar. Caracas, Venezuela: Fundación Biblioteca Ayacucho.

Ortiz, R. (2014). Universalismo/diversidad. Contradicciones de la modernidad-mundo. Buenos Aires, Argentina: Prometeo.

Pelfini, A. (2013). Del cosmopolitismo "atlántico" al cosmopolitismo minimalista. La subjetivación de América Latina en una modernidad plural. Devenires. Revista de Filosofía y Filosofía de la Cultura, XIV (28), 13-38.

Restrepo, E. (2014). Interculturalidad en cuestión. Cerramientos y potencialidades. Ámbito de Encuentros, 7 (1), 9-30.

Rodríguez, G. (2010). Transcultura y Hegemonía. Inguruak Revista Vasca de Sociología y Ciencia Política. Monográfico Especial. Sociedad e innovación en el siglo XXI. Bilbao.

Rogers, R. (2006). From cultural exchange to transculturation. A review and reconceptualization of cultural appropriation. Communication Theory, 16 (4), 474-503.

Ruffino, R. y Chinzari, S. (2014). Where the border stands. From war ambulance to intercultural exchanges. Italia: HOEPLI.

Santos, M. (2009). Preparar ciudadanos en una era global ¿puede ayudar la educación intercultural? Avances en supervisión educativa. Revista de la Asociación de Inspectores de Educación de España, 11.

Schmidt-Welle, F. (2011). Transculturación, heterogeneidad y ciudadanía cultural. Algunas consideraciones. En F. Schmidt-Welle (coord.), Multiculturalismo, transculturación, 
heterogeneidad, poscolonialismo. Hacia una crítica de la interculturalidad (pp. 41-60). México: Herder.

Sorrells, K. (2010). Re-imagining intercultural communication in the context of globalization. En T. Nakayama y R. T. Halualani (eds.), The handbook of critical intercultural communication (pp. 171-189). Oxford, Reino Unido: Wiley-Blackwell.

Sorrells, K. y Nakagawa, G. (2008). Intercultural communication praxis and the struggle for social responsibility and social justice. En O. Swartz (ed.), Transformative communication studies. Culture, hierarchy, and the human condition (pp. 17-43). Lancaster: Troubador Publishing.

Thurlow, C., Killick D., Parry, M. y Phipps, A. (2001). 'I don't have one-it's just normal'. Young teenagers' ideas about 'culture'. critical transcultural communication awareness and the exoticisation of self. En D. Killick y M. Parry (eds.), Mapping the territory: the poetics and praxis of languages and intercultural communication (pp. 111-124). Glasgow: Glasgow French and German Publications.

Tubino, F. (2005). La interculturalidad crítica como proyecto ético-político. Lima, Perú: Centro de Desarrollo Étnico.

UNESCO. (2006). Directrices de La UNESCO sobre la educación intercultural.

- - - . (2010). Education for intercultural understanding. reorienting teacher education to address sustainable development. guidelines and tools. Bangkok, Thailand: UNESCO.

-_-_. (2012). La iniciativa mundial la educación ante todo. Una iniciativa del Secretario General de las Naciones Unidas. Education First. UNESCO.

- - - . (2014a). Global citizenship education. Preparing Learners for the challengers of the tweinty-first century. Paris, Francia: UNESCO.

-__—. (2014b). Learning to live together. Education policies and realities in the asia-pacific. Bangkok, Thailand: UNESCO.

-_-_. (2015). Education for All 2000-2015. Achievements and challenges; EFA global monitoring report. Paris, Francia: UNESCO Publishing. 
Vodopivec, N. (2012). Challenging global citizenship through interculturality. Crossing borders and practicing solidarity. Critical Literacy. Theories and Practices, 6 (1).

Walsh, C. (2011). Etnoeducación e interculturalidad en perspectiva decolonial. Lima, Perú: Centro de Desarrollo Étnico.

Welsch, W. (1999). Transculturality - the puzzling form of culture today. En M. Featherstone y S. Lash (ed.), Space of culture. City, nation, world (pp. 194-213). Londres, Reino Unido: Sage.

————. (2011). ¿Qué es la transculturalidad? En F. Schmidt-Welle (coord.), Multiculturalismo, transculturación, heterogeneidad, poscolonialismo. Hacia una crítica de la Interculturalidad (pp. 11-40). México: Herder.

Zizek, S. (1998). Multiculturalismo o la lógica cultural del capitalismo multinacional. En F. Jameson, y S. Zizek, Estudios culturales. Reflexiones sobre el multiculturalismo (pp. 137188). Buenos Aires, Argentina: Paidós. 